copied into RNA, and there also exist systems in which RNA molecules can be amplified through a DNA intermediate by a combination of reverse transcriptase and polymerase. Thus, immense populations can be handled and molecules with various properties can be iteratively selected. Two chapters, by L. Gold et al. and by J. Szostak and A. Ellington, discuss this burgeoning field of artificial genetics. Gold has searched RNA sequence space for ligands and he (and others) has discovered RNA molecules that bind not only to proteins that normally have nucleic acid ligands but also to others such as thrombin and nerve growth factor that do not. It still remains to be seen how easy it is to pass from this sequence space to that of the simple heterocyclic molecules favoured by the pharmaceutical industry.

In a coda to his paper, Gold has some stringent remarks to make about scientists (like myself) who view nucleic acids "as strings of information rather than sources of interesting globular ligands". In his enthusiasm to simulate "experiments done billions of years ago" (with nerve growth factor?) he has forgotten that there may also be a need to simulate modern systems as well and his particular objections can be easily dealt with. RNA molecules provide the easiest implementation for exploration in combinatorial chemistry, but there are other technologies which can and will be used to explore test-tube molecular evolution.

Although, in principle, these discoveries with RNA molecules, both natural and artificial, narrow the gap between primitive genetic systems in which function and information were carried and copied within the same molecule and the modern bilingual systems, they do not help much with the origin of life itself. In a sobering first chapter, G. Joyce and L. Orgel point out that there are still enormous difficulties in going from a mixture of purine and pyrimidine bases to any kind of selfreplicating system. We seem to have crossed the gap between molecular biology and genetics, but that between chemistry and molecular biology remains wide and deep. In fact, some people have worried that there may not have been enough time to have done it all in our solar system. Crick refers in his foreword to his hypothesis of directed panspermia which gains time by having the early steps going on somewhere else in the Universe followed by the transport to Earth of a population of microorganisms a few billion years ago to continue the good work here. Thus, when we come to the pre-RNA world, we will have to decide not only what to look for but also where to look for it.

Sydney Brenner is in the Department of Medicine, University of Cambridge School of Clinical Medicine, Addenbrooke's Hospital, Hills Road, Cambridge CB2 2QQ, UK.

\section{Toxic barb}

Alastair Hay

General and Applied Toxicology, Edited By Bryan Ballentyne, Timothy Marrs and Paul Turner. Stockton/Macmillan Press: 1993. Pp. 1,456. \$295, £195.

ASSESSING a general text on toxicology is not that far removed from assessing the potential risk of a particular chemical. It is necessary to know what each does. How specific is the available information? Are underlying mechanisms explained and dissected? Further considerations include the treatment of general information about target organs involved, effects on the environment and, it is to be hoped, discussions about species differences.

General and Applied Toxicology meets

\section{IMAGE UNAVAILABLE FOR COPYRIGHT REASONS}

The military applications of toxicology - a victim of chemical warfare.

these criteria. Aimed at students of the subject, the book is a mine of information for both the generalist interested in, for example, the principles underlying toxicity testing, and for the specialist concerned about gases released during combustion and the damage they can cause.

The book is intentionally wide-ranging. Well-known researchers have contributed review chapters on the subject of their speciality. Published in two volumes, the book is further subdivided into sections dealing with basic concepts such as study design, haematology and clinical chemistry; toxicity by contact route; target organs; specialist toxicology, which includes chapters on mutagenicity, epigenetic carcinogenesis and neonatal toxicology; regulatory toxicology; and, finally, single issues such as the role of epidemiology, or the toxicology of pesticides, che- mical warfare agents and food additives.

One particularly pleasing feature of the book is the crispness of the chapters. Most are very well written and concise as a result, presumably, of a firm editorial hand. This conciseness enables the authors to be wide-ranging in their coverage of topics and, for some readers, sections may be all too brief. The chapters are, however, extremely well referenced and more interested specialists will find plenty to guide them to other research sources.

The effects of explosive devices are not addressed specifically, although chapters on combustion toxicology and chemicals used in warfare to asphyxiate or to poison steer the book around the area of the military applications of the subject. For some scientific researchers, explosive devices may have represented all too real a hazard. As targets of animal rights activists and with growing public concern about the welfare of animals, toxicologists have had their share of bad press. The criticisms have also brought rewards. Increasing emphasis is now placed on the use by researchers of well-validated in vitro systems of testing.

Such tests have revolutionized the in$\stackrel{5}{\circ}$ vestigation of mutagens and potential car-

$\Sigma$ cinogens and may do the same when applied to many other areas of toxicology through the use of cell cultures from specific organs. These may in time be useful predictive models for the effects of chemicals on target organs. In vitro systems and the ethical issues that have to be dealt with by toxicologists are also aired in the book.

General and Applied Toxicology tends to emphasize the pragmatic approach to the regulation of chemicals and drugs exemplified by countries in the European Union, and by the Food and Drug Administration in the United States. Chapters on regulation review the processes involved and, according to the editors, regulatory toxicology is becoming "increasingly dominant, though sometimes scientifically suspect". This comment is a barb directed, no doubt, at some regulatory agencies, such as the US Environmental Protection Agency, which use predictive models for estimating cancer risk for substances at very low doses. Such models can vary by as much as $10^{6}$ in their prediction of the risk involved, and are extrapolated from data obtained from high-dosage tests. These models are not used in the United Kingdom for reaching regulatory decisions.

Although far from cheap, General and Applied Toxicology helps to fill a particular niche in the toxicology literature. Purchasers are likely to judge that their money has been well spent.

Alastair Hay is in the Chemical Pathology Department, University of Leeds, Leeds LS2 9JZ, UK. 